\title{
The MacGuffin Block Cipher Algorithm
}

\author{
Matt Blaze ${ }^{1}$ and Bruce Schneier ${ }^{2}$ \\ 1 AT\&T Bell Laboratories \\ 101 Crawfords Corner Road, Holmdel, NJ 07733 USA \\ maboresearch.att.com \\ 2 Counterpane Systems \\ 730 Fair Oaks Avenue, Oak Park, IL 70302 USA \\ schneierochinet.com
}

\begin{abstract}
This paper introduces MacGuffin, a 64 bit "codebook" block cipher. Many of its characteristics (block size, application domain, performance and implementation structure) are similar to those of the U.S. Data Encryption Standard (DES). It is based on a Feistel network, in which the cleartext is split into two sides with one side repeatedly modified according to a keyed function of the other. Previous block ciphers of this design, such as DES, operate on equal length sides. MacGuffin is unusual in that it is based on a generalized unbalanced Feistel network (GUFN) in which each round of the cipher modifies only 16 bits according to a function of the other 48 . We describe the general characteristics of MacGuffin architecture and implementation and give a complete specification for the 32-round, 128-bit key version of the cipher.
\end{abstract}

\section{Introduction}

Feistel ciphers [1] operate by alternately encrypting the bits in one "side" of their input based on a keyed non-linear function of the bits in the other. This is done repeatedly, for a fixed number of "rounds". It is believed that, when iterated over sufficiently many rounds, even relatively simple non-linear functions can provide high security. Traditionally, such ciphers split their input block evenly about the middle; a 64 bit cipher would operate on two 32 bit internal blocks, swapping the "left" (the target block) and "right" (the control block) sides with each round. Several important block ciphers, including DES [3], are built upon this structure. We say these ciphers are based on balanced Feistel networks (BFNs), since both sides are of equal length.

This paper describes a block cipher, called MacGuffin, that is based on a variant of this structure, the generalized unbalanced Feistel network (GUFN), in which the target and control blocks need not be of equal length ${ }^{3}$. GUFNs, especially those in which the target block is smaller than the control block,

\footnotetext{
${ }^{3}$ Several cryptographic hash functions, such as MD5 [6] and SHA [5], employ an
} unbalanced structure similar in some respects to a GUFN. 
appear to have a number of attractive properties for cipher design, particularly with respect to the design of the non-linear function. The principles underlying GUFNs are discussed in [7].

As its name suggests, MacGuffin is intended primarily as a catalyst for discussion and analysis. We believe it may also prove a practical, high security block cipher suitable for general use as an alternative to DES. It operates on 64 bit blocks of data, with an internal structure containing a 16 bit target block and a 48 bit control block (" 48 on 16 ", in the notation of [7]). In principle, almost any length key and any number of rounds may be used, although we specify 32 rounds and a 128 bit key as "standard".

\section{Architecture}

We have been conservative in most aspects of MacGuffin's design, isolating most of its novel features to those parts of the design related to its unbalanced structure. As such, much of our design is adapted directly from DES. We hope that the many similarities between DES and MacGuffin will invite analysis of their differences.

Basically, the input cipherblock is partitioned into four 16 bit words, from left to right. In each round, the three rightmost words comprise the control block and are bitwise exclusive-ORed (XORed) with three words derived from the key. These 48 bits are then split eight ways according to a fixed permutation to provide input to eight functions of six bits (the "S-boxes"), each producing two bits of output. The $16 \mathrm{~S}$-box output bits are then XORed, according to another fixed permutation, with the bits in the leftmost (target) word. Finally, the leftmost word is rotated into the rightmost position. The cipher can be reversed by a similar process, with the key derived bits applied in reverse order.

\subsection{Design Principles}

Because each round operates on only half as many bits as in a BFN (16 as opposed to 32 ), we use 32 rounds, twice as many as in DES, in our standard version. Because there are twice as many rounds, however, there are also a total of twice as many key bits XORed with the control blocks. These bits are obtained from the 128 bit key with the key expansion function described in the next section.

We adapt our S-boxes directly from those of DES. The eight DES S-boxes each produce four bits of output. Since we require only two bits from each (for a total of 16 bits), we use only the "outer" two output bits from each S-box.

In each round, each control block bit is XORed with one derived key bit and provides one input to exactly one S-box. There is no "expansion" permutation, since the number of control bits equals the number of S-box inputs. The control bits are mapped 1: 1 to S-box inputs according to a fixed permutation. This permutation was designed so that each $S$-box receives two of its six inputs from each of the three registers in the control block. 
S-box outputs are distributed across the 16 target bits. No S-box output goes to a bit position that is used as a direct input to itself in the next four rounds.

Observe that each of the three control registers contains bits produced in a different round of the cipher, and that each encrypted bit provides input to three different S-boxes (in the next three rounds), before it is encrypted again.

The cipher is designed for implementation in either hardware or software. Permutations were chosen to minimize the number of shift and mask operations and to allow time/memory optimizations in a software implementation.

\section{Algorithm Description}

\subsection{Data Structures and Notation}

We use the following notation:

$\oplus$ represents a 16 bit bitwise exclusive-OR (XOR) operation.

$\leftarrow$ is the conventional assignment operator, except as noted below.

$w, x, y, z \leftarrow i$ copies the data from 64 bit interface $i$, from lowest to highest bit position, into 16 bit registers $w, x, y$ and $z$, respectively.

$i \leftarrow w, x, y, z$ copies the bits from 16 bit registers $w, x, y$ and $z$, respectively into interface $i$, from lowest to highest bit position.

$s, t, u, v \leftarrow w, x, y, z$ copies $w, x, y$ and $z$ to $s, t, u$ and $v$, respectively, in parallel (e.g., $x, y \leftarrow y, x$ swaps $x$ and $y$ ).

$w \Leftarrow F(x, y, z)$ selects, according to a fixed permutation, bits from $x, y$ and $z$ as input to function $F$, storing the function output in bits of $w$, selected according to a fixed permutation.

The cipher employs the following internal structures:

$I_{0 \ldots 63}, O_{0 \ldots 63}$ are the 64 bit external input and output interfaces.

left, $a, b, c, t$ are 16 bit registers on which all cryptographic operations are performed. $r_{0}$ represents the least significant bit of $r, r_{15}$ the most significant. $k_{0 \ldots 127}$ is a 128 bit secret key parameter.

$K[0 \ldots 31,0 \ldots 2]$ is a $32 \times 3$ table of 16 bit words containing an expansion of $k$, as explained below.

\subsection{S-boxes and Permutations}

Nonlinearity in the encryption and key setup processes is provided primarily through eight functions, or "S-boxes", denoted $S_{1} \ldots S_{8}$, each taking six bits of input selected from the $a, b$ and $c$ registers and producing two bits of output (which are XORed into the left register).

Inputs to each S-box are selected uniquely from the $a, b$ and $c$ registers, as specified in Table 1 . (In this table, input bit 0 is the least significant bit.) Outputs from each S-box are distributed across the 16 bit target block as specified in Table 2. Each S-box is defined as a $64 \times 2$ bit mapping of input values to outputs, as given in Table 3 . 


\begin{tabular}{|c|cccccc|}
\hline & \multicolumn{7}{|c|}{ Input Bit } \\
S-box & 0 & 1 & 2 & 3 & 4 & 5 \\
\hline$S_{1}$ & $a_{2}$ & $a_{5}$ & $b_{6}$ & $b_{9}$ & $c_{11}$ & $c_{13}$ \\
$S_{2}$ & $a_{1}$ & $a_{4}$ & $b_{7}$ & $b_{10}$ & $c_{8}$ & $c_{14}$ \\
$S_{3}$ & $a_{3}$ & $a_{6}$ & $b_{8}$ & $b_{13}$ & $c_{0}$ & $c_{15}$ \\
$S_{4}$ & $a_{12}$ & $a_{14}$ & $b_{1}$ & $b_{2}$ & $c_{4}$ & $c_{10}$ \\
$S_{5}$ & $a_{0}$ & $a_{10}$ & $b_{3}$ & $b_{14}$ & $c_{6}$ & $c_{12}$ \\
$S_{6}$ & $a_{7}$ & $a_{8}$ & $b_{12}$ & $b_{15}$ & $c_{1}$ & $c_{5}$ \\
$S_{7}$ & $a_{9}$ & $a_{15}$ & $b_{5}$ & $b_{11}$ & $c_{2}$ & $c_{7}$ \\
$S_{8}$ & $a_{11}$ & $a_{13}$ & $b_{0}$ & $b_{4}$ & $c_{3}$ & $c_{9}$ \\
\hline
\end{tabular}

Table 1. S-Box Input Permutation

\begin{tabular}{|c|cc|}
\hline & \multicolumn{3}{|c|}{ Output Bit } \\
S-box & 0 & 1 \\
\hline$S_{1}$ & $t_{0}$ & $t_{1}$ \\
$S_{2}$ & $t_{2}$ & $t_{3}$ \\
$S_{3}$ & $t_{4}$ & $t_{5}$ \\
$S_{4}$ & $t_{6}$ & $t_{7}$ \\
$S_{5}$ & $t_{8}$ & $t_{9}$ \\
$S_{6}$ & $t_{10}$ & $t_{11}$ \\
$S_{7}$ & $t_{12}$ & $t_{13}$ \\
$S_{8}$ & $t_{14}$ & $t_{15}$ \\
\hline
\end{tabular}

Table 2. S-Box Output Permutation

\subsection{Key Setup}

Each round of the cipher uses the secret key parameter to perturb the S-boxes by bitwise XOR against the S-box inputs. Each round thus requires 48 key bits. To convert the 128 bit $k$ parameter to a sequence of 48 bit values for each round (the $K$ table), MacGuffin uses an iterated version of its own block encryption function. See Figure 1.

\subsection{Block Encryption}

Block encryption is defined in Figure 2.

\subsection{Block Decryption}

Block decryption is similar to block encryption, and is defined in Figure 3. 


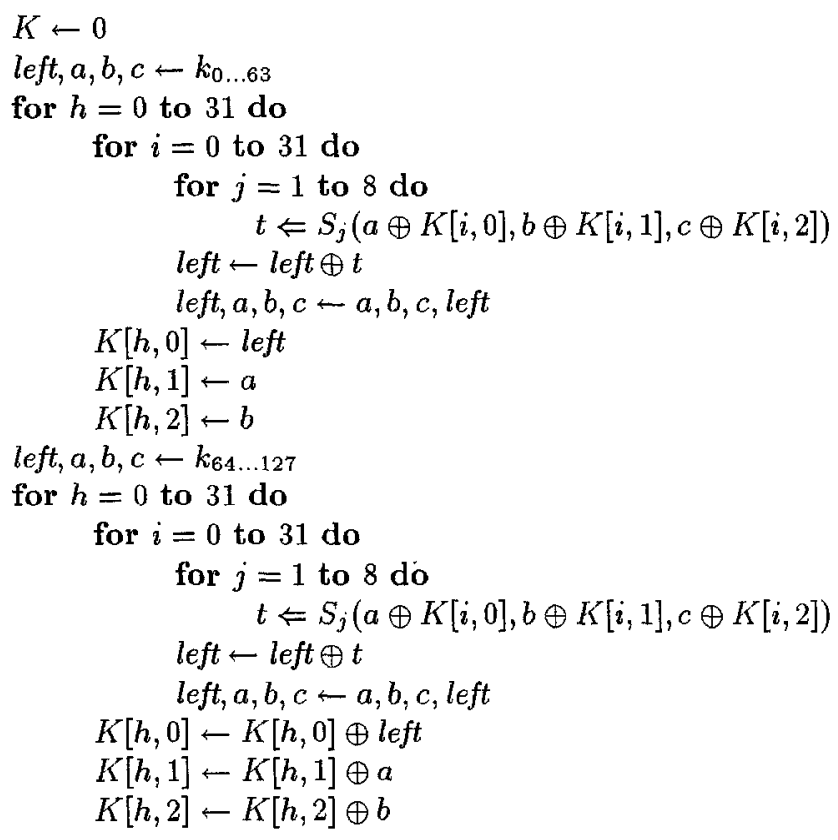

Fig. 1. MacGuffin Key Setup

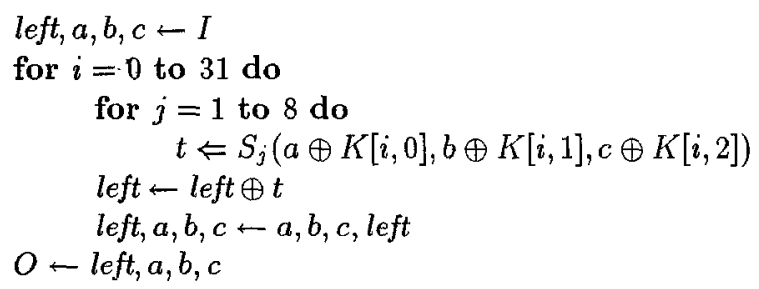

Fig. 2. MacGuffin Block Encryption

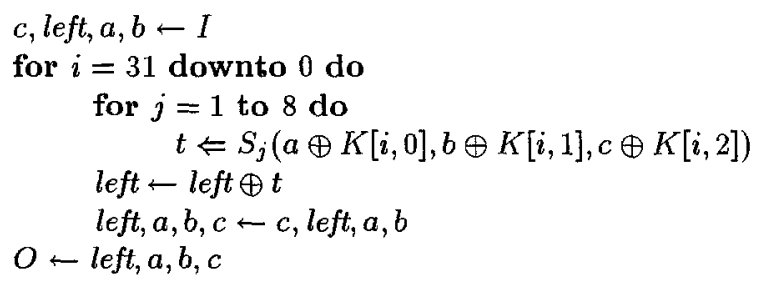

Fig. 3. MacGuffin Block Decryption 
$S_{1}$

20033110023033211220022313310112

03122220300301313123311212201003

$S_{2}$

31132021033012023210013220031321

03221231210330101320210230110233

$S_{3}$

23013023011030121032211232030321

31020330203312013013022113212012

$S_{4}$

13322311000330211001201231220233

21033000223113323310112312012002

$S_{5}$

02230012102133012110133231032230

03021231213210233033201302100121

$S_{6}$

22132030310203210031130220131132

30213012032123121302012110303203

$S_{7}$

03300321300321321221311210230210

10033332211012212331002302103102

$\frac{S_{8}}{31032302023131102231102310022310}$

10310211302222030302233031111023

Table 3. MacGuffin S-Boxes

\section{Implementation, Performance and Applications}

Feistel ciphers, with their many permutation operations and table lookups, are particularly well suited to hardware implementation. Because permutations in hardware are "free" (they are implemented with simple connections), and because S-box lookups can occur in parallel, each round can be implemented with conventional modern hardware in two clock cycles.

Software implementations of Feistel ciphers on general-purpose computers are typically much slower than their hardware counterparts, since the S-boxes must be evaluated in sequence and bit permutations must be simulated with 
shifts, ANDs, ORs and other operators. Depending on the specific permutations and S-box structures, however, many of these operations can be made faster with table lookups and by combining several operations into one.

The permutations in MacGuffin have been designed explicitly to permit software optimization. First, the six inputs to each S-box are from different bits from each of the $a, b$ and $c$ registers, allowing the three registers to be masked and ORed together (without individual shifting) for a single lookup for each S-box. Furthermore, for each S-box there is a unique "mate" S-box with which it shares no common inputs. This allows the eight S-boxes to be "paired off" and looked up two at a time with a single $2^{16}$ entry table containing the combined outputs of both S-boxes. (The pairs are $S_{1} S_{2}, S_{3} S_{4}, S_{5} S_{7}$ and $S_{6} S_{8}$ ).

An optimized software implementation (given in the Appendix) of 32 round MacGuffin runs at close to the speed of optimized 16 round DES in software. An implementation on a $486 / 66$ processor has a bandwidth of about $1.5 \mathrm{Mbps}$; a reasonable DES implementation [2] on the same processor runs at 2.1Mbps.

The MacGuffin interface is similar to that of DES (except for the larger keyspace). It can be used with the standard "FIPS-81" modes of operation[4]. Note that key setup is an explicitly time consuming process. This is intended to discourage exhaustive search of poorly chosen keys. In an implementation where rapid selection among many keys is required (such as a packet-based network security protocol) the 1536 bit expanded key may be passed directly as the cryptovariable.

Experiments with MacGuffin are detailed in [7].

While we believe the GUFN structure is superior to the conventional BFN cipher structure, much more discussion and analysis is required before we can recommend its use for protecting sensitive data. We encourage attacks against MacGuffin in particular and the GUFN structure in general.

\section{References}

1. H. Feistel. Cryptography and Computer Privacy. Scientific American, May 1973.

2. J. Lacy, D.P. Mitchell, and W.M. Schell. CryptoLib: Cryptography in Software. Proceedings of USENIX Security Symposium IV, October 1993.

3. National Bureau of Standards. Data Encryption Standard, Federal Information Processing Standards Publication 46, US Government Printing Office, Washington, D.C., 1977.

4. National Bureau of Standards. Data Encryption Standard Modes of Operation, Federal Information Processing Standards Publication 81, US Government Printing Office, Washington, D.C., 1980.

5. National Institute for Standards and Technology. Secure Hash Standard. Federal Information Processing Standard Publication 180, US Government Printing Office, April 1993.

6. R. Rivest. The MD5 Message Digest Algorithm. RFC 1321, IETF, April 1992.

7. B. Schneier and M. Blaze. Unbalanced Feistel Network Block Ciphers. To appear, 1994. 


\section{Appendix: Optimized C Language Implementation}

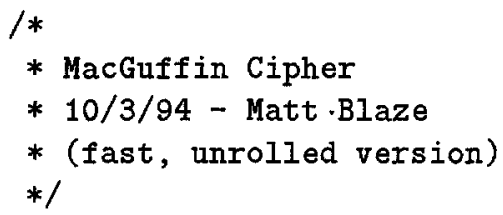

/* the 8 s-boxes, expanded to put the output bits in the right

* places. note that these are the des s-boxes (in left-right,

* not canonical, order), but with only the "onter" two output

* bits. */

unsigned short sboxes [8] [64] $=\{$

$1 * 0$ (S1) */

$\{0 \times 0002,0 \times 0000,0 \times 0000,0 \times 0003,0 \times 0003,0 \times 0001,0 \times 0001,0 \times 0000$, $0 \times 0000,0 \times 0002,0 \times 0003,0 \times 0000,0 \times 0003,0 \times 0003,0 \times 0002,0 \times 0001$, $0 \times 0001,0 \times 0002,0 \times 0002,0 \times 0000,0 \times 0000,0 \times 0002,0 \times 0002,0 \times 0003$, $0 \times 0001,0 \times 0003,0 \times 0003,0 \times 0001,0 \times 0000,0 \times 0001,0 \times 0001,0 \times 0002$, $0 \times 0000,0 \times 0003,0 \times 0001,0 \times 0002,0 \times 0002,0 \times 0002,0 \times 0002,0 \times 0000$, $0 \times 0003,0 \times 0000,0 \times 0000,0 \times 0003,0 \times 0000,0 \times 0001,0 \times 0003,0 \times 0001$, $0 \times 0003,0 \times 0001,0 \times 0002,0 \times 0003,0 \times 0003,0 \times 0001,0 \times 0001,0 \times 0002$, $0 \times 0001,0 \times 0002,0 \times 0002,0 \times 0000,0 \times 0001,0 \times 0000,0 \times 0000,0 \times 0003\}$, $1 * 1$ (S2) */

$\{0 \times 000 c, 0 \times 0004,0 \times 0004,0 \times 000 c, 0 \times 0008,0 \times 0000,0 \times 0008,0 \times 0004$, $0 \times 0000,0 \times 000 \mathrm{c}, 0 \times 000 \mathrm{c}, 0 \times 0000,0 \times 0004,0 \times 0008,0 \times 0000,0 \times 0008$, $0 \times 000 \mathrm{c}, 0 \times 0008,0 \times 0004,0 \times 0000,0 \times 0000,0 \times 0004,0 \times 000 \mathrm{c}, 0 \times 0008$, $0 \times 0008,0 \times 0000,0 \times 0000,0 \times 000 \mathrm{c}, 0 \times 0004,0 \times 000 \mathrm{c}, 0 \times 0008,0 \times 0004$, $0 \times 0000,0 \times 000 \mathrm{c}, 0 \times 0008,0 \times 0008,0 \times 0004,0 \times 0008,0 \times 000 \mathrm{c}, 0 \times 0004$, $0 \times 0008,0 \times 0004,0 \times 0000,0 \times 000 \mathrm{c}, 0 \times 000 \mathrm{c}, 0 \times 0000,0 \times 0004,0 \times 0000$, $0 \times 0004,0 \times 000 \mathrm{c}, 0 \times 0008,0 \times 0000,0 \times 0008,0 \times 0004,0 \times 0000,0 \times 0008$, $0 \times 000 \mathrm{c}, 0 \times 0000,0 \times 0004,0 \times 0004,0 \times 0000,0 \times 0008,0 \times 000 \mathrm{c}, 0 \times 000 \mathrm{c}\}$, /*2 $2(\mathrm{~S} 3) * /$

$\{0 \times 0020,0 \times 0030,0 \times 0000,0 \times 0010,0 \times 0030,0 \times 0000,0 \times 0020,0 \times 0030$, $0 \times 0000,0 \times 0010,0 \times 0010,0 \times 0000,0 \times 0030,0 \times 0000,0 \times 0010,0 \times 0020$, $0 \times 0010,0 \times 0000,0 \times 0030,0 \times 0020,0 \times 0020,0 \times 0010,0 \times 0010,0 \times 0020$, $0 \times 0030,0 \times 0020,0 \times 0000,0 \times 0030,0 \times 0000,0 \times 0030,0 \times 0020,0 \times 0010$, 
$0 \times 0030,0 \times 0010,0 \times 0000,0 \times 0020,0 \times 0000,0 \times 0030,0 \times 0030,0 \times 0000$, $0 \times 0020,0 \times 0000,0 \times 0030,0 \times 0030,0 \times 0010,0 \times 0020,0 \times 0000,0 \times 0010$, $0 \times 0030,0 \times 0000,0 \times 0010,0 \times 0030,0 \times 0000,0 \times 0020,0 \times 0020,0 \times 0010$, $0 \times 0010,0 \times 0030,0 \times 0020,0 \times 0010,0 \times 0020,0 \times 0000,0 \times 0010,0 \times 0020\}$, $1 * 3(\mathrm{~S} 4) * 1$

$\{0 \times 0040,0 \times 00 c 0,0 \times 00 c 0,0 \times 0080,0 \times 0080,0 \times 00 c 0,0 \times 0040,0 \times 0040$, $0 \times 0000,0 \times 0000,0 \times 0000,0 \times 00 c 0,0 \times 00 c 0,0 \times 0000,0 \times 0080,0 \times 0040$, $0 \times 0040,0 \times 0000,0 \times 0000,0 \times 0040,0 \times 0080,0 \times 0000,0 \times 0040,0 \times 0080$, $0 \times 00 \mathrm{c0}, 0 \times 0040,0 \times 0080,0 \times 0080,0 \times 0000,0 \times 0080,0 \times 00 \mathrm{c0}, 0 \times 00 \mathrm{c0}$, $0 \times 0080,0 \times 0040,0 \times 0000,0 \times 00<0,0 \times 00 c 0,0 \times 0000,0 \times 0000,0 \times 0000$, $0 \times 0080,0 \times 0080,0 \times 00 c 0,0 \times 0040,0 \times 0040,0 \times 00 c 0,0 \times 00 c 0,0 \times 0080$, $0 \times 00 \mathrm{co}, 0 \times 00 \mathrm{c0}, 0 \times 0040,0 \times 0000,0 \times 0040,0 \times 0040,0 \times 0080,0 \times 00 \mathrm{co}$, $0 \times 0040,0 \times 0080,0 \times 0000,0 \times 0040,0 \times 0080,0 \times 0000,0 \times 0000,0 \times 0080\}$, $1 * 4(\mathrm{~S} 5) * 1$

$\{0 \times 0000,0 \times 0200,0 \times 0200,0 \times 0300,0 \times 0000,0 \times 0000,0 \times 0100,0 \times 0200$, $0 \times 0100,0 \times 0000,0 \times 0200,0 \times 0100,0 \times 0300,0 \times 0300,0 \times 0000,0 \times 0100$, $0 \times 0200,0 \times 0100,0 \times 0100,0 \times 0000,0 \times 0100,0 \times 0300,0 \times 0300,0 \times 0200$, $0 \times 0300,0 \times 0100,0 \times 0000,0 \times 0300,0 \times 0200,0 \times 0200,0 \times 0300,0 \times 0000$, $0 \times 0000,0 \times 0300,0 \times 0000,0 \times 0200,0 \times 0100,0 \times 0200,0 \times 0300,0 \times 0100$, $0 \times 0200,0 \times 0100,0 \times 0300,0 \times 0200,0 \times 0100,0 \times 0000,0 \times 0200,0 \times 0300$, $0 \times 0300,0 \times 0000,0 \times 0300,0 \times 0300,0 \times 0200,0 \times 0000,0 \times 0100,0 \times 0300$, $0 \times 0000,0 \times 0200,0 \times 0100,0 \times 0000,0 \times 0000,0 \times 0100,0 \times 0200,0 \times 0100\}$, /* $5(\mathrm{~S} 6) * /$

$\{0 \times 0800,0 \times 0800,0 \times 0400,0 \times 0 c 00,0 \times 0800,0 \times 0000,0 \times 0<00,0 \times 0000$, $0 \times 0 c 00,0 \times 0400,0 \times 0000,0 \times 0800,0 \times 0000,0 \times 0 \times 00,0 \times 0800,0 \times 0400$, $0 \times 0000,0 \times 0000,0 \times 0<00,0 \times 0400,0 \times 0400,0 \times 0<00,0 \times 0000,0 \times 0800$, $0 \times 0800,0 \times 0000,0 \times 0400,0 \times 0 c 00,0 \times 0400,0 \times 0400,0 \times 0 c 00,0 \times 0800$, $0 \times 0 c 00,0 \times 0000,0 \times 0800,0 \times 0400,0 \times 0 \times 00,0 \times 0000,0 \times 0400,0 \times 0800$, $0 \times 0000,0 \times 0 \mathrm{c} 00,0 \times 0800,0 \times 0400,0 \times 0800,0 \times 0 \mathrm{c} 00,0 \times 0400,0 \times 0800$, $0 \times 0400,0 \times 0 \times 00,0 \times 0000,0 \times 0800,0 \times 0000,0 \times 0400,0 \times 0800,0 \times 0400$, $0 \times 0400,0 \times 0000,0 \times 0 c 00,0 \times 0000,0 \times 0 \times 00,0 \times 0800,0 \times 0000,0 \times 0 c 00\}$, $1 * 6(\mathrm{~S} 7) * 1$

$\{0 \times 0000,0 \times 3000,0 \times 3000,0 \times 0000,0 \times 0000,0 \times 3000,0 \times 2000,0 \times 1000$, $0 \times 3000,0 \times 0000,0 \times 0000,0 \times 3000,0 \times 2000,0 \times 1000,0 \times 3000,0 \times 2000$, $0 \times 1000,0 \times 2000,0 \times 2000,0 \times 1000,0 \times 3000,0 \times 1000,0 \times 1000,0 \times 2000$, $0 \times 1000,0 \times 0000,0 \times 2000,0 \times 3000,0 \times 0000,0 \times 2000,0 \times 1000,0 \times 0000$, $0 \times 1000,0 \times 0000,0 \times 0000,0 \times 3000,0 \times 3000,0 \times 3000,0 \times 3000,0 \times 2000$, $0 \times 2000,0 \times 1000,0 \times 1000,0 \times 0000,0 \times 1000,0 \times 2000,0 \times 2000,0 \times 1000$, $0 \times 2000,0 \times 3000,0 \times 3000,0 \times 1000,0 \times 0000,0 \times 0000,0 \times 2000,0 \times 3000$, $0 \times 0000,0 \times 2000,0 \times 1000,0 \times 0000,0 \times 3000,0 \times 1000,0 \times 0000,0 \times 2000\}$, $1 * 7(\mathrm{~S} 8) * 1$

$\{0 \times 0000,0 \times 4000,0 \times 0000,0 \times c 000,0 \times 8000,0 x c 000,0 \times 0000,0 \times 8000$, $0 \times 0000,0 \times 8000,0 \times 1000,0 \times 4000,0 \times 10000,0 \times 4000,0 \times 4000,0 \times 0000$, $0 \times 8000,0 \times 8000,0 \times 1000,0 \times 4000,0 \times 4000,0 \times 0000,0 \times 8000,0 \times 0000$, $0 \times 4000,0 \times 0000,0 \times 0000,0 \times 8000,0 \times 8000,0 \times 0000,0 \times 4000,0 \times 0000$, 
$0 \times 4000,0 \times 0000,0 \times 0000,0 \times 4000,0 \times 0000,0 \times 8000,0 \times 4000,0 \times 4000$, $0 \times 0000,0 \times 0000,0 \times 8000,0 \times 8000,0 \times 8000,0 \times 8000,0 \times 0000,0 \times 0000$, $0 \times 0000,0 \times 0000,0 \times 0000,0 \times 8000,0 \times 8000,0 \times 0000,0 \times 0000,0 \times 0000$, $0 \times 1000,0 \times 4000,0 \times 4000,0 \times 4000,0 \times 4000,0 \times 0000,0 \times 8000,0 \times 0000\}$ \};

/* table of s-box outputs, expanded for 16 bit input.

* this one table includes all 8 sboxes - just mask off

* the output bits not in use. */

unsigned short stable[TSIZE];

/* we exploit two features of the s-box input \& output perms -

* first, each s-box uses as input two different bits from each

* of the three registers in the right side, and, second,

* for each s-box there is another-sbox with no common input bits

* between them. therefore we can lookup two s-box outputs in one

* probe of the table. just mask off the approprate input bits

* in the table below for each of the three registers and OR

* together for the table lookup index.

* these masks are also available below in \#defines, for better

* lookup speed in unrolled loops. */

unsigned short lookupmasks [4] [3] $=\{$

/*a $, \quad \mathrm{b}, \quad \mathrm{c} * /$
$\{0 \times 0036,0 \times 06 \mathrm{co}, 0 \times 6900\}, / * s 1+s 2 * /$
$\{0 \times 5048,0 \times 2106,0 \times 8411\}, / * s 3+s 4 * /$
$\{0 \times 8601,0 \times 4828,0 \times 10 \mathrm{c} 4\}, / * s 5+s 7 * /$
$\{0 \times 2980,0 \times 9011,0 \times 022 a\}\} ; / * s 6+s 8 * /$

/* this table contains the corresponding output masks for the

* lookup procedure mentioned above.

* (similarly available below in \#defines). */

unsigned short outputmasks [4] $=\{$

$0 \times 000 f / * s 1+s 2 * /$, $0 x 00 f 0 / * s 3+s 4 * /$, $0 \times 3300 / * s 5+s 7 * /, 0 x \operatorname{cc00} / * s 6+s 8 * /\}$

/* input and output lookup masks (see above) */

$/ * \operatorname{sits} 2 * /$

\#define INOO

\#define IN01

$0 \times 0036$

\#define INO2 0x6900

\#define OUTO 0x000f

$1 * s 3+s 4 * 1$

\#define IN10 $0 \times 5048$

\#define IN11 0x2106

\#define IN12 0x8411 


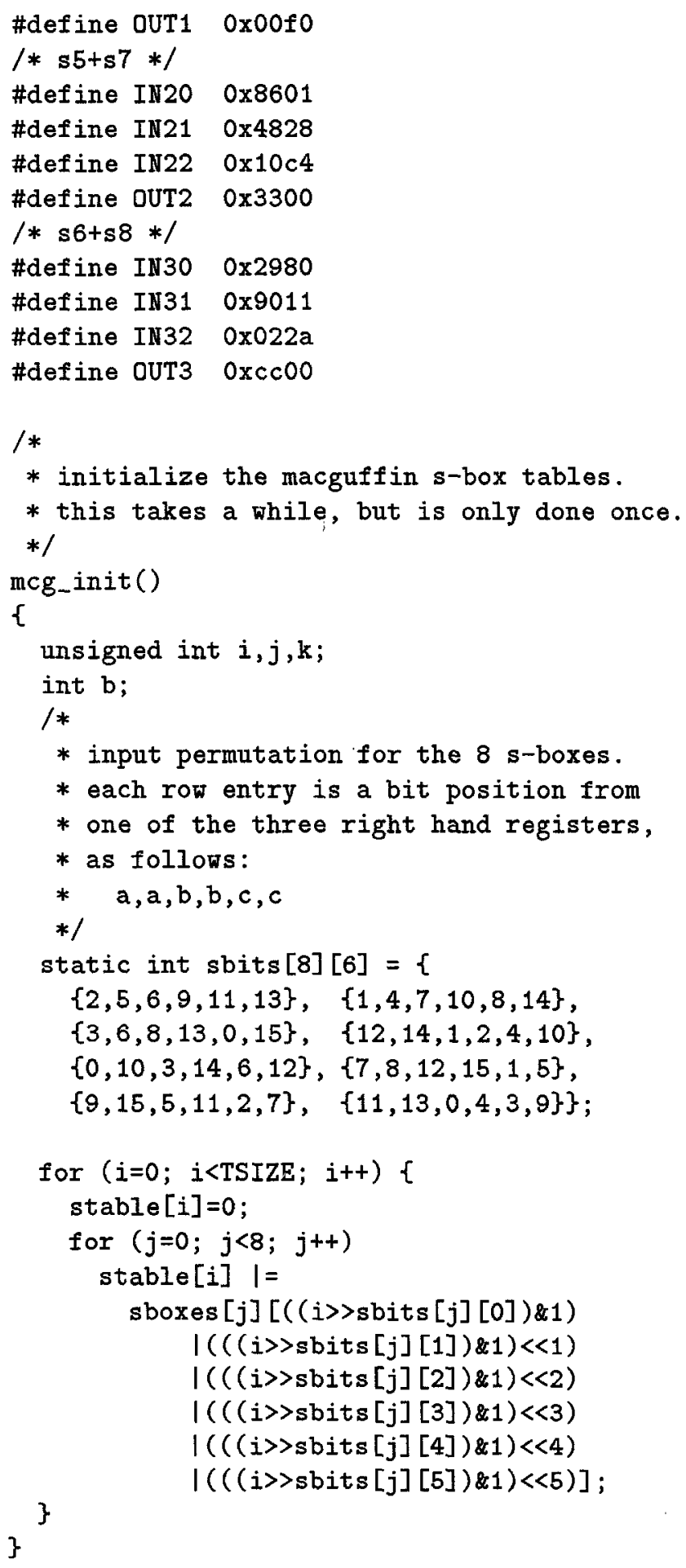




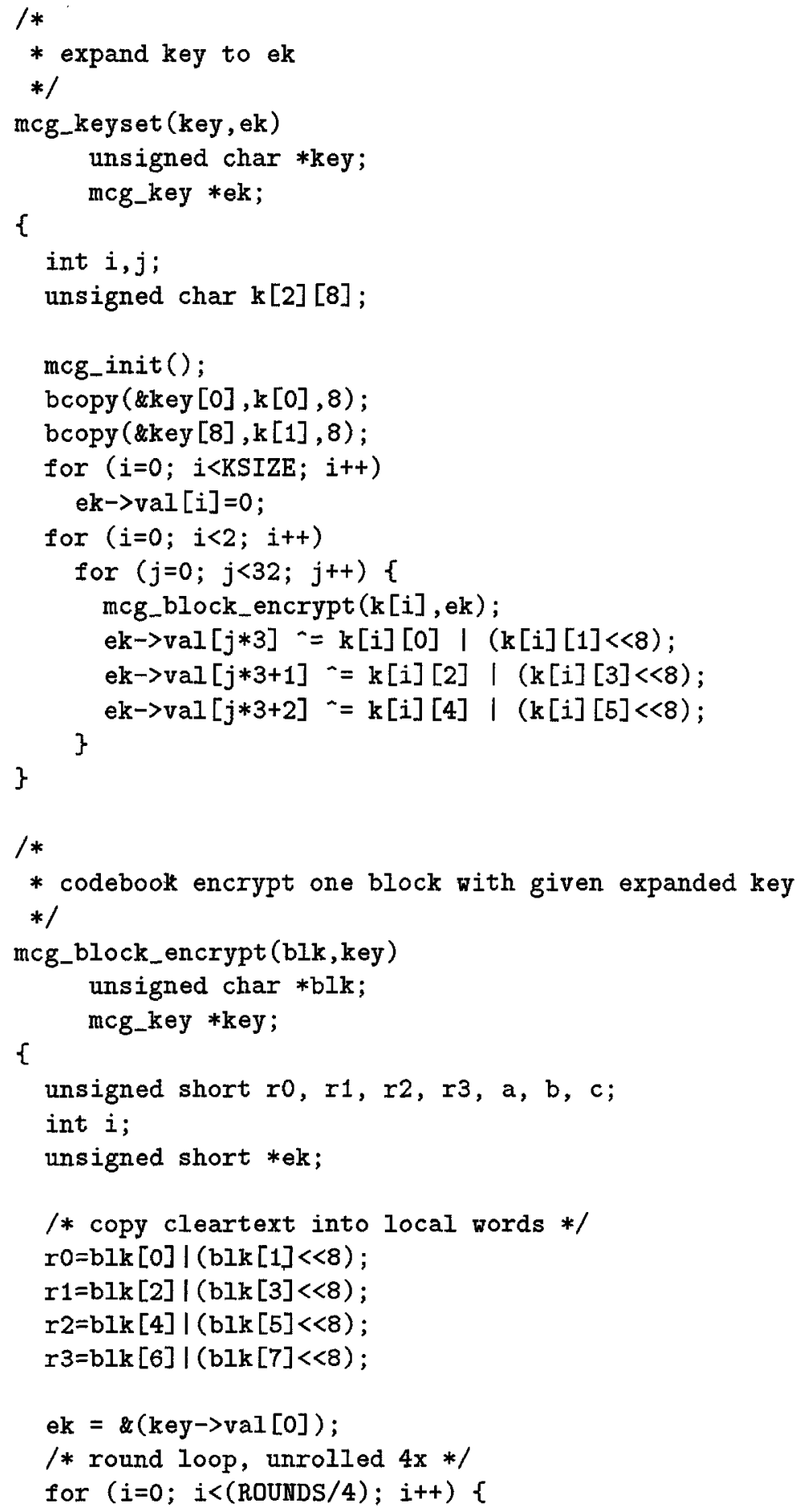




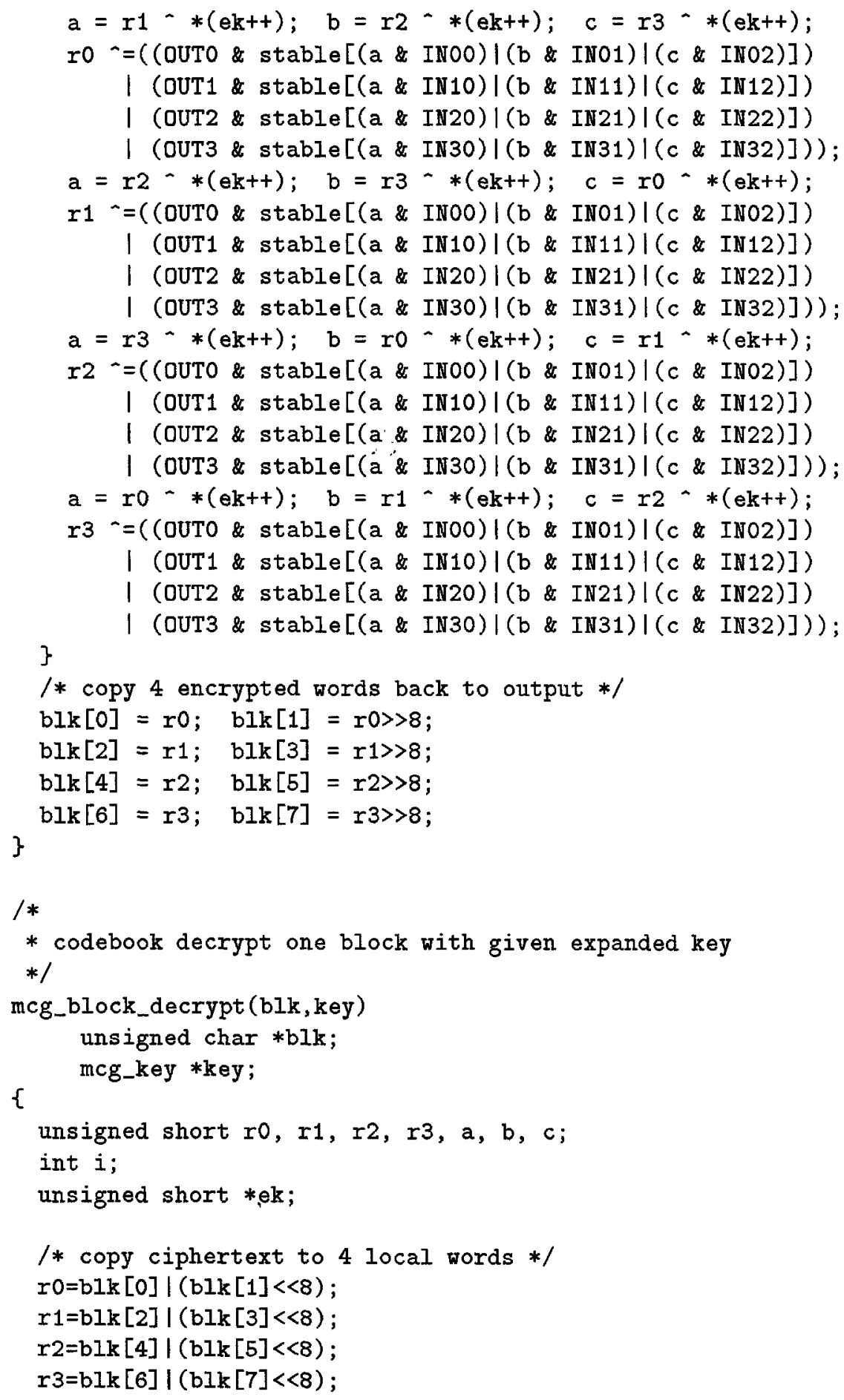




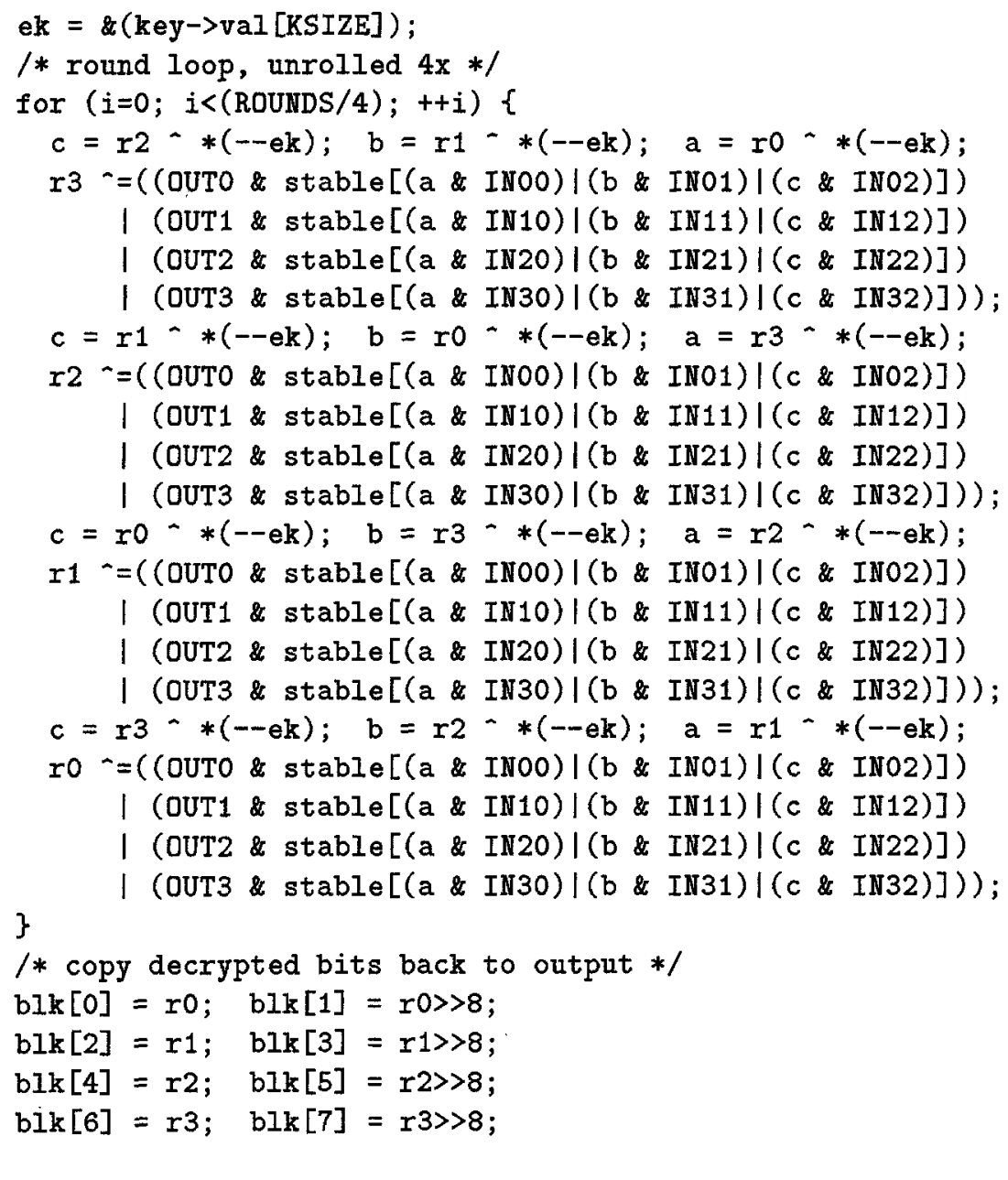

